\title{
Comparación de la eficiencia técnica de los sistemas de salud en países pertenecientes a la oms
}

\author{
ARMANDO NEVÁREZ-SidA \\ Patricia Constantino-Casas \\ FERnando García-Contreras*
}

\section{Resumen}

Con base en información de insumos y productos de la Organización Mundial de la Salud (OMs) de 2002, se estimó y comparó (con un método determinístico no paramétrico: Data Envelopment Analysis, técnica DEA) la eficiencia técnica de 191 países con respecto a los resultados en salud y su relación con la distribución del ingreso y el ingreso per cápita. Se propusieron dos modelos con diferentes variables dependientes. Los países se clasificaron por ingreso per cápita en cuatro categorías y se compararon respecto de su eficiencia técnica. Se encontró correlación negativa entre el nivel de eficiencia técnica e ingreso per cápita. Las comparaciones mostraron que los países con menor ingreso tuvieron mayor eficiencia técnica.

Palabras clave: eficiencia técnica, sistemas de salud, producción de salud, DEA.

\begin{abstract}
Based on information from the World Health Organisation about supplies and products in 2002, we estimated and compared the technical efficiency of 191 countries (using a non-parametric deterministic method: the Data Envelopment Analysis (DEA) technique) taking into consideration the results on health and its relationship with income distribution and per capita income. We have proposed two models with different dependent variables. The countries were classified by their per capita income into four categories and they were compared with respect to the technical efficiency obtained. We found a negative correlation between the level of technical efficiency and the per capita income. The comparisons showed that those countries with a lower income obtained a grater technical efficiency.
\end{abstract}

Keywords: technical efficiency, health systems, health production, DEA.

* Todos los autores están adscritos al Instituto Mexicano del Seguro Social, México. Correo-e de contacto: armando.nevarez@ imss.gob.mx. 


\section{Introducción}

Todos los países invierten parte de sus recursos en los sistemas de salud de acuerdo con sus prioridades y políticas. En 2001, los países afiliados a la Organización Mundial de la Salud (OMS) gastaron, en promedio, $5.8 \%$ de su producto interno bruto (РІB) en salud (wHO, 2002). La magnitud per cápita de esas inversiones se relaciona de manera directa con el grado de riqueza de cada país, su población y la agenda de políticas nacionales de salud. Sin embargo, independientemente de la magnitud de inversión en salud existe interés por conocer si los recursos empleados se utilizan de la mejor forma.

Algunos estudios estiman la eficiencia de los sistemas de salud con diferentes metodologías en distintos grupos de países. David Evans y colaboradores (2001) midieron la eficiencia estimando la relación entre los niveles de salud de la población (esperanza de vida saludable) y los insumos utilizados para producirla (gasto en salud per cápita y promedio de años de escolaridad de la población adulta). Estos autores emplearon métodos econométricos en una muestra de 191 países con información de 1993 a 1997. La conclusión fue que el incremento en los recursos de salud en países pobres y la utilización más eficiente de los recursos en países ricos, son las mejores alternativas para mejorar los resultados de salud en cada caso.

Hugh Gravelle y colaboradores (2002) utilizaron distintas definiciones de eficiencia y métodos de estimación para corroborar los resultados obtenidos por Evans y colaboradores (2001) sobre la eficiencia de los sistemas nacionales de salud. Informaron que el ordenamiento de países y la magnitud de eficiencia fueron sensibles a la forma como ésta es definida y a los modelos de análisis.

Donna Retzlaff Roberts y colaboradores (2004) emplearon la metodología propuesta en el presente estudio para calcular la eficiencia técnica de los países de la Organización para la Cooperación y el Desarrollo Económico (OCDE). Concluyeron, entre otras cosas, que Estados Unidos podría disminuir los insumos que emplea sin afectar su esperanza de vida.

De manera sistemática se ha encontrado una relación entre ingreso, distribución del ingreso y variables de salud. Jennifer Mellor y Jeffrey Milyo (2001), analizando países desarrollados, encontraron una correlación positiva entre el coeficiente de Gini y la mortalidad infantil, y una correlación negativa entre la esperanza de vida y el coeficiente de Gini. En relación con el gasto en 
salud y su impacto en la mortalidad infantil, Deon Filmer y Lan Pritchett (1999) comentaron que éste no es un determinante poderoso, sino que existen otros factores como ingreso per capita, desigualdad en la distribución del ingreso, educación femenina, nivel de fragmentación étnica y religión predominante. Richard Wilkinson y Kate Pickett (2006) revisaron 155 artículos sobre la relación de ingreso y resultados en salud, y en $70 \%$ de ellos se describe que los niveles menores de salud se asocian con los mayores niveles de desigualdad en el ingreso.

A pesar de la relación que existe entre el ingreso, su distribución y los resultados en salud, no está suficientemente documentada la relación entre el ingreso, o las medidas de distribución del ingreso, y el desempeño de los sistemas de salud. Este último representado por una medida de eficiencia que relaciona uno o más resultados en salud con algunos de los insumos requeridos para su producción.

Es fundamental conocer si las inversiones en salud proporcionan los mejores resultados; es decir, si los recursos se emplean de manera eficiente. El objetivo del presente estudio es estimar y comparar la eficiencia técnica de 191 países pertenecientes a la oms respecto de sus resultados en salud y su relación con la distribución del ingreso y el ingreso per cápita en la muestra de países analizada por la OMS (WHO, 2000).

\section{Material y métodos}

La eficiencia técnica en salud, definida como la relación entre insumos y productos en el área de la salud (Palmer y Torgerson, 1999), se estimó en cada país con un método determinístico no paramétrico que utiliza técnicas de programación lineal, conocido como deA (Data Envelopment Analysis) (Cooper et al., 2000).

Mediante esta técnica se definió la frontera de mejor práctica, también denominada tecnología de referencia o frontera de producción. Esta frontera se construyó mediante la identificación de aquellos países que producen una cantidad determinada de productos con el menor número de insumos. Cuanto más cercana se situó una observación de la frontera, más próximo se encontró ese país de alcanzar la eficiencia. Si se localizó una observación en la frontera, entonces ese país se consideró eficiente.

La técnica DEA permite construir una frontera de producción con cualquier número de insumos y de productos. En la figura I se ilustra cómo se construye la frontera de mejor práctica en el caso de un solo insumo y un solo producto (Fare et al., 1994). La 
línea recta que surge desde el origen representa la tecnología bajo rendimientos constantes a escala (RCE). En el mismo diagrama se observa la frontera de referencia para rendimientos no crecientes a escala (RNCE) que va de $0 \mathrm{a} A B$ y la línea horizontal a partir de B. En el caso de la tecnología con rendimientos variables a escala (RVE), la frontera de referencia se inicia en el punto cuya abscisa es $\mathrm{x}^{\mathrm{t}}$, de ahí continúa a $\mathrm{AB}$ y al segmento horizontal desde $\mathrm{B}$ (figura I).

\section{Figura I}

Construcción de la tecnología de referencia

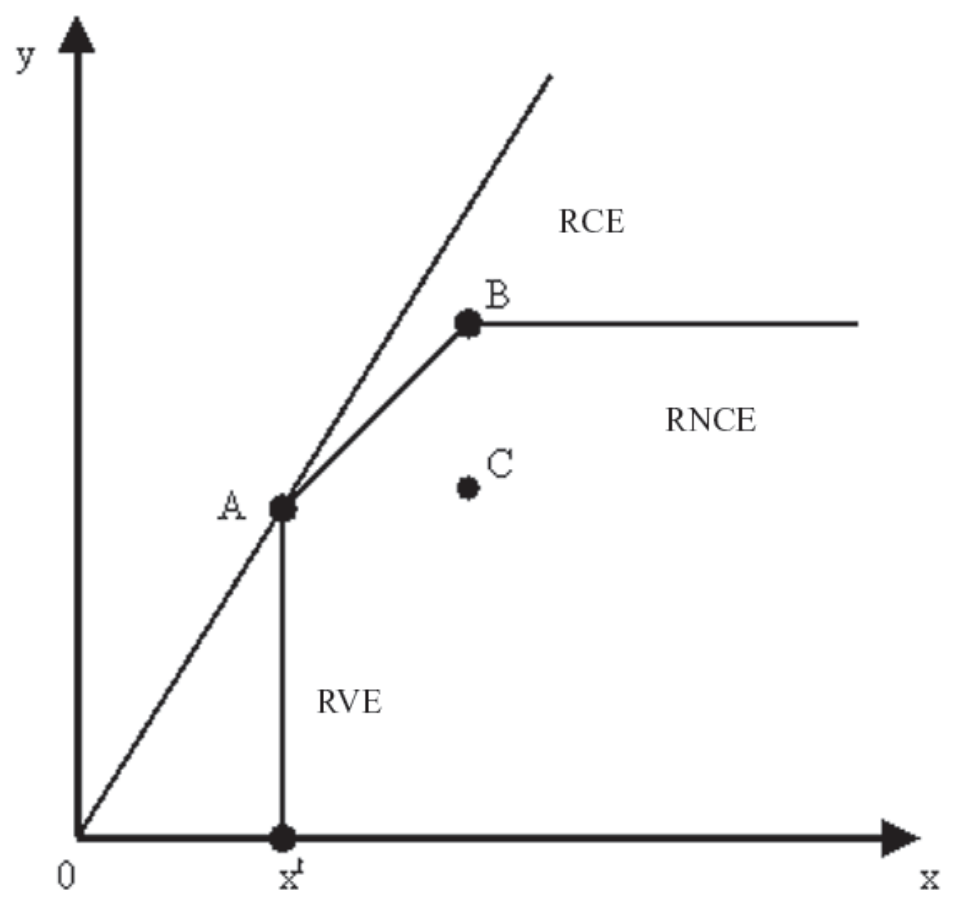

Fuente: Elaborada con base en Fare et al. (1994).

Un procedimiento es técnicamente eficiente (Bitran y Block, 1992) si los insumos de producción se combinan de forma que se alcance el máximo producto. La eficiencia económica extiende el concepto de eficiencia técnica para tomar en cuenta los precios relativos de los insumos de producción. Así, un procedimiento es económicamente eficiente si, y sólo si, los insumos son combinados para producir un nivel dado de producto al costo 
mínimo, por lo que no todas las combinaciones técnicamente eficientes lo serán también desde el punto de vista económico.

Ambos tipos de eficiencia se pueden determinar mediante la técnica DEA. Sin embargo, en el presente artículo solamente se calculó la eficiencia técnica debido a la disponibilidad de información. Para fines de simplificación, la eficiencia técnica se denomina eficiencia, y se entiende como la proporción en la que los insumos pueden ser reducidos para obtener el mismo producto.

El escenario base asumió fuerte disposición de insumos; es decir, si se incrementan los insumos, el producto no puede decrecer, por lo que no tiene congestión (no se produce con una cantidad excesiva de insumos). También se asumieron rendimientos no crecientes a escala, por lo que un incremento proporcional en los insumos implicó un aumento en el producto en la misma o menor proporción.

El problema a resolver consistió en optimizar el producto sujeto a restricciones, para el caso de rendimientos no crecientes (N) y fuerte disposición de insumos (S). Se asume que hay $\mathrm{k}=1, \ldots$, $\mathrm{K}$ países que usan $\mathrm{n}=1, \ldots, \mathrm{N}$ insumos $x_{k n}$. Los insumos se emplean para producir $\mathrm{m}=1, \ldots, \mathrm{M}$ productos $y_{k n}$. La frontera de referencia se construyó como:

$$
\begin{aligned}
& P(x / N, S)=\left\{\left(y_{1}, \ldots, y_{M}\right):\right. \\
& \sum_{k=1}^{K} z_{k} y_{k m} \geq y_{m}, m=1, \ldots, M, y_{m}=\sum_{k=1}^{K} y_{k m} \\
& \sum_{k=1}^{K} z_{k} x_{k n} \leq x_{n}, n=1, \ldots, N, x_{n}=\sum_{k=1}^{K} y_{k n} \\
& \left.\sum_{k=1}^{K} z_{k} \leq 1, z_{k} \geq 0, k=1, \ldots, K\right\} .
\end{aligned}
$$

Donde $\mathrm{z}_{\mathrm{k}}, \mathrm{k}=1, \ldots, \mathrm{K}$ son variables de intensidad. Si la segunda restricción se convierte en igualdad, se modela una disposición débil de insumos. Para modelar rendimientos variables, se incluyó una igualdad en la tercera restricción; es decir, la sumatoria de las variables de intensidad para cada país debe ser igual a uno.

Con fines de simplificación, se definieron las categorías de eficiencia alta, media y baja (ineficiencia). Los países con alta eficiencia fueron aquéllos que se encontraron en la frontera de mejor práctica. 
Las dos categorías restantes correspondieron a países con eficiencia media e ineficiencia. El punto de corte fue el promedio de eficiencia de todos los países en cada uno de los modelos.

Para realizar el análisis de sensibilidad se efectuaron algunos cambios en los supuestos del modelo base. Uno de estos cambios fue asumir una disposición débil de insumos haciendo posible una reducción de insumos sin que esto implique necesariamente una reducción en el producto.

Se relajó también el supuesto de rendimientos no crecientes a escala para permitir rendimientos variables a escala. Si se supone que el gasto en salud se incrementa de manera constante año tras año, ello no implica que los niveles de salud de la población se incrementen también en forma constante, dado que existen otras variables involucradas y un nivel máximo de salud alcanzable, y a partir de este punto cualquier incremento en los insumos implicará un mayor gasto y una reducción en la eficiencia.

Para alcanzar un determinado nivel de salud, ya sea individual o para una región o país, los factores que intervienen son muchos. Uno de los más relevantes serían las acciones del sistema de salud en su conjunto; sin embargo, si se careciera de él, el nivel de salud no sería cero (Murray y Frenk, 1999), por lo que su importancia es relativa. El nivel de salud bajo el supuesto de que no existe sistema de salud fue calculado por Evans y colaboradores (2001) utilizando observaciones de 25 países para 1908 (en promedio) y extrapolando esas relaciones para 1997. Aunque desde el punto de vista teórico sería deseable contar con la estimación del nivel de salud de cada país sin su sistema de salud para corregir el cálculo de eficiencia, consideramos que no es posible obtener esa información, y que (como piensan otros autores, como Gravelle et al., 2002) resulta irrelevante sustraer el nivel de salud sin sistema ( $s a-$ lud mínima), ya que ello impacta tanto en el numerador como en el denominador sin modificar el cálculo de la eficiencia.

Por otro lado, el producto teórico, nivel de salud, no es fácil de determinar pues presenta numerosos indicadores con diferentes grados de agregación y no es fácilmente observable, por lo que se utilizaron medidas indirectas de ese producto.

Para tener una aproximación más apropiada de la variable dependiente se utilizaron los siguientes indicadores de producto:

- Esperanza de vida al nacer (Ev): se definió como el número de años que una persona espera vivir al nacer si los niveles de mortalidad permanecen constantes en un lugar y tiempo específicos. 
- Mortalidad infantil (MI): es el número de muertes en niños menores de un año de edad ocurridas durante un periodo de un año expresado por 1,000 nacidos vivos.

- Esperanza de vida saludable: este indicador se calcula con base en la esperanza de vida al nacer, pero incluye un ajuste por el tiempo estimado en el que se tiene una salud pobre; es decir, equivale al número de años con salud completa (Mathers et al., 2000).

Las dos primeras variables (EV y MI) son de importancia en cuanto a indicadores de desempeño nacional e internacional. Sin embargo, como lo deseable es vivir el mayor número de años con una buena salud, se incluyó la esperanza de vida saludable. En función de las tres variables de producto definidas previamente se desarrollaron dos modelos: en el primero, los productos que explican el desempeño de un sistema de salud son la esperanza de vida y la mortalidad infantil, mientras que en el segundo la variable de producto fue la esperanza de vida saludable al nacer. La ventaja del primer modelo es que los indicadores no se agrupan en un índice por la subjetividad para la elección de ponderadores apropiados (Smith, 2002).

Por el lado de los insumos, idealmente se deberían incluir todos aquellos factores que inciden en la obtención de un determinado estado de salud. Sin embargo, debido a que intervienen múltiples factores y muchos de ellos no son fácilmente cuantificables, se consideraron solamente dos: uno ligado de manera directa con el desempeño del sistema de salud (gasto en salud per cápita), y el segundo, más relacionado con las características socioeconómicas de la población de un país (PIB per cápita), como educación, vivienda y alimentación. Estos dos indicadores se definieron de la siguiente forma:

- Gasto en salud per cápita (GSP): es el gasto total en salud de cada país entre su población. Para hacer estos datos comparables se expresaron en dólares ajustados por paridad del poder de compra (PPP).

- Producto interno bruto per cápita (PIBP): conjunto de todos los bienes y servicios producidos en el interior de un país, ya sea por nacionales o extranjeros, expresado en términos monetarios y dividido por la población del país; datos también expresados en dólares ajustados por PpP. 
Todas estas variables se obtuvieron directamente de los informes que proporciona la OMs sobre los países miembros (WHOsIs, 2005). A diferencia de Evans y colaboradores (2001), que no incluyeron el PIB per cápita por presentar problemas de correlación tanto con el gasto en salud per cápita como con la educación, la utilización de la técnica DEA permite eliminar problemas de multicolinealidad, por lo que en el presente estudio se incluyó dicha variable.

Para fines de la presentación de los datos e interpretación de los resultados, se clasificaron los países con base en su ingreso per cápita en cuatro grupos tomando como límites los percentiles 25,50 y 75 : con ingreso bajo, medio bajo, medio y alto (véase Anexo).

\section{Resultados}

Se obtuvieron cuatro indicadores de eficiencia para cada uno de los 191 países, dos por cada uno de los modelos y, dentro de éstos, uno corresponde a rendimientos no crecientes a escala y fuerte disposición de insumos, y el otro a rendimientos variables a escala y disposición débil de insumos.

Los resultados de eficiencia del primer modelo se presentan en el cuadro 1. Los productos del sistema de salud considerados son la esperanza de vida y la mortalidad infantil.

El grupo de países de bajos ingresos tuvo más integrantes con eficiencia alta en sus sistemas de salud, mientras que los países

\section{Cuadro 1}

\section{Eficiencia por tipo de país en el modelo 1} (porcentaje)

\begin{tabular}{lccc}
\hline Tipo de país & Ineficiente & Eficiencia media & Eficiencia alta \\
\hline \multicolumn{2}{l}{ Rendimientos no crecientes } & & \\
Ingreso bajo & 43.75 & 43.75 & 12.50 \\
Ingreso medio-bajo & 44.68 & 44.68 & 10.64 \\
Ingreso medio & 87.76 & 10.20 & 2.04 \\
Ingreso alto & 51.06 & 40.43 & 8.51 \\
Rendimientos variables & & & \\
Ingreso bajo & 50.00 & 29.17 & 20.83 \\
Ingreso medio-bajo & 42.55 & 42.55 & 14.89 \\
Ingreso medio & 83.67 & 6.12 & 10.20 \\
Ingreso alto & 51.06 & 31.91 & 17.02 \\
\hline
\end{tabular}

Fuente: Cálculos propios con base en información de la oms. 
con ingresos medios fueron los más ineficientes. Se encontró, en general, que a mayor ingreso se obtiene menor eficiencia, ya que la rho de Spearman mostró una correlación negativa $(r h o=-0.27$; $p<0.0001$ ).

En relación con la distribución de países por tipo de ingreso y eficiencia bajo rendimientos variables a escala, en el cuadro 1 se observa que, de forma independiente al grupo de ingreso, una mayor proporción de países se ubicó dentro de la frontera de producción, por lo que, según la calificación, se les atribuye una eficiencia alta.

Bajo rendimientos variables y disposición débil de insumos, 30 países se ubicaron sobre la frontera de mejor práctica. Lo anterior representó un número muy superior a los 16 que se encontraban en esa situación bajo rendimientos no crecientes a escala y disposición fuerte de insumos. La correlación entre el nivel de ingreso y la eficiencia con rendimientos variables mostró un valor $r$ ho $=-0.127(p=0.015)$.

El segundo modelo difiere del primero en que la variable de resultado del sistema de salud es la esperanza de vida saludable. En este modelo se obtuvo un coeficiente de correlación rho de Spearman entre el ingreso per cápita y la eficiencia bajo rendimientos no crecientes de $-0.431(p<0.0001)$, mientras que con rendimientos variables a escala el valor de $r$ o fue de -0.32 ( $p<$ $0.0001)$. Estos datos muestran que existe una correlación importante y negativa entre el nivel de ingreso per cápita y la eficiencia de los sistemas de salud.

En el cuadro 2 se presenta el número de países que mejoraron o empeoraron su medida de eficiencia por el cambio de modelo. Los países que permanecieron igual fueron, en su mayoría, aquellos que se ubicaron en la frontera de mejor práctica de forma independiente al tipo de rendimientos a escala y/o variable(s) de desempeño (entre ellos Japón, Malta, Sri Lanka y Singapur).

\section{Cuadro 2 \\ Países que cambiaron su desempeño en función del modelo}

\begin{tabular}{lccccrc}
\hline Tipo de país & $\begin{array}{c}\text { Rendimientos no } \\
\text { Peor }\end{array}$ & Igual & Mejor & \multicolumn{3}{c}{ Rendimientos variables } \\
& 32 & 6 & 10 & 30 & 11 & 7 \\
\hline Ingreso bajo & 23 & 3 & 21 & 17 & 5 & 25 \\
Ingreso medio-bajo & 23 & 2 & 26 & 17 & 6 & 26 \\
Ingreso medio & 21 & 2 & 35 & 11 & 5 & 31 \\
Ingreso alto & 10 & 2 & 35 &
\end{tabular}

Fuente: Cálculos propios con base en información de la oms. 
Los resultados de eficiencia para cada uno de los países se modifican de acuerdo con las especificaciones de los modelos, y estas diferencias son estadísticamente significativas (prueba de Wilcoxon $p<0.0001$ ). Los países que fueron eficientes en el modelo 1 (con rendimientos no crecientes) son poco sensibles a cambios en los modelos y supuestos de disposición de insumos y rendimientos. Algunos países de América Latina cuya eficiencia fue poco sensible a los cambios en los modelos fueron México, Brasil, República Dominicana, Costa Rica y Panamá.

La eficiencia de acuerdo con el grupo de países por tipo de ingreso en ambos modelos muestra que, salvo el grupo de ingresos altos, a menor ingreso se tiene mayor eficiencia. Como se puede observar en la figura II, el grupo de países con ingresos altos tiene un comportamiento diferente al resto. El grupo que mostró la menor eficiencia fue el de ingresos medios.

Figura II

\section{Gráfica comparativa por ingresos}

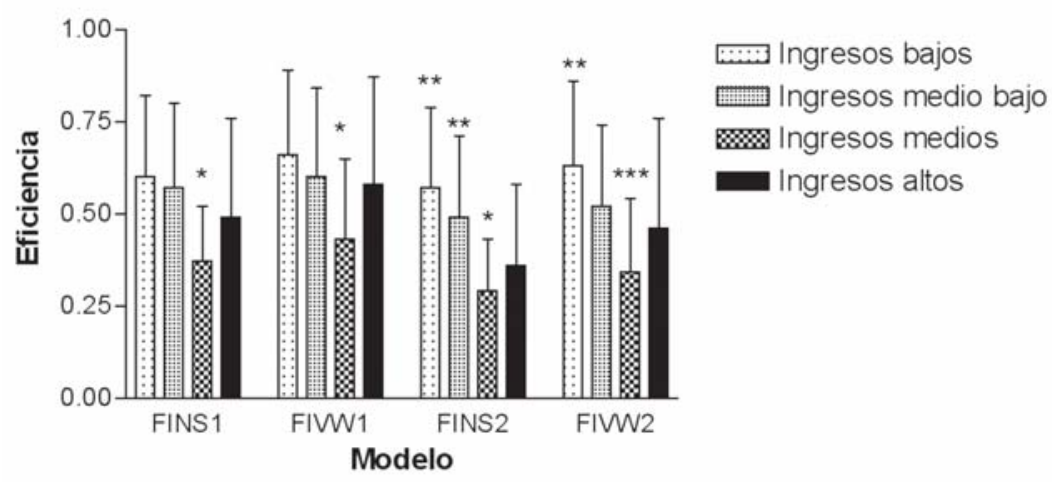

FINS1. Modelo 1, rendimientos no crecientes y fuerte disposicion de insumos

FIVW1. Modelo 1, rendimientos variables y debil disposicion de insumos

FINS2. Modelo 2, rendimientos no crecientes y fuerte disposición de insumos

FNW2. Modelo 2, rendimientos variables y debil disposición de insumos

- Diferente al resto de los grupos $p<0.05$; ANOVA 1 via; Bonferroni.

"Diferente con el grupo de ingresos attos: $p<0.05$; ANOV A 1 via; Bonferroni.

-.. Diferente con grupo de ingresos bajos y medio bajos: $p<0.05$; ANOV A 1 via: Bonferroni.

Debido a que las mediciones de eficiencia del sistema de salud dependen de manera importante de causas ajenas al mismo, como son la educación y la cultura de la población (expresadas de manera indirecta por el ingreso per cápita), una medida de distribución del ingreso estaría asociada de manera negativa con los resultados en eficiencia alcanzados por los sistemas de salud 
(Tangcharoensathien y Lertiendumrong, 2000). Es decir, los países con una distribución del ingreso más homogénea tendrán mejores resultados en salud que aquellos países que globalmente tengan mayores ingresos pero en donde la distribución de éstos sea más desigual.

La correlación entre la distribución del ingreso y la eficiencia de los sistemas de salud se corroboró mediante el coeficiente de correlación rho de Spearman. Se encontró, para el primer modelo, una correlación negativa de la eficiencia con el coeficiente de Gini con un valor $r h o=-0.269(p=0.003)$ en rendimientos no crecientes, mientras que con rendimientos variables fue de -0.246 $(p=0.006)$. En el segundo modelo la eficiencia no mostró correlación estadística significativa con el coeficiente de Gini. Los resultados anteriores se obtuvieron con datos de 124 países, debido a que el resto no tenía la información necesaria para realizar el análisis.

\section{Conclusiones}

Se encontró correlación significativa entre el desempeño de los sistemas de salud y el nivel de ingreso de los países estudiados. Aunque dicha correlación fue muy baja, estos valores son compatibles con los datos obtenidos por Akira Babazono y Alan Hillman (1994) y por Deon Filmer y Lant Pritchett (1999). En ambos modelos, e independientemente del nivel de ingreso de determinado país, potencialmente siempre existirá un margen para obtener mejores resultados de salud utilizando más eficientemente los recursos disponibles sin tener que destinar necesariamente mayores recursos a ese sector. Esto extiende el resultado obtenido por Howard Oxley y Maitland MacFarlan (1994) para los países integrantes de la OCDE.

Aunque la eficiencia de los sistemas de salud se correlacionó de manera inversa con el coeficiente de Gini, ya que los países con similares inversiones en salud obtuvieron una calificación de su eficiencia mejor si presentaban menor desigualdad en el ingreso, ello no es más que un resultado preliminar y con limitaciones. En la literatura relacionada con el tema existe controversia (Bartley, 2004), por lo que se requiere invertir en la obtención de información de calidad y en el desarrollo de más investigación al respecto.

De acuerdo con lo desarrollado en el presente documento, más de 50\% de los países que integran la oms tienen sistemas de salud ineficientes, por lo que las modificaciones orientadas a incrementar 
su eficiencia derivarán en cambios positivos y sustanciales en los resultados de salud (entre ellos, Guinea Ecuatorial, Sudáfrica, Botswana, Swazilandia y Liberia). Cuarenta por ciento de los países que tienen una eficiencia media pueden mejorar sus indicadores de resultados en salud de manera modesta con cambios encaminados a la eficiencia. De acuerdo con los resultados obtenidos en los distintos modelos, países como Estados Unidos o Suiza pueden disminuir en cierto rango los insumos empleados sin afectar la esperanza de vida, la mortalidad infantil y la esperanza de vida saludable.

Contrario a lo obtenido por Evans y colaboradores (2001), en el presente estudio se encontró que China tiene un buen desempeño en comparación con países con similares niveles de desarrollo. Por su parte, Sri Lanka mostró un sistema de salud comparativamente eficiente; estos resultados son consistentes con los de otros autores (Halstead et al., 1985; Hsiao, 2000).

La ubicación de 19 países dentro de la frontera de mejor práctica no implica que su eficiencia no se pueda incrementar de un periodo a otro. Es decir, en este periodo son ellos los que utilizan de manera más eficiente sus combinaciones de insumos para obtener el máximo producto, pero éste es solamente el máximo de un periodo, no el máximo global que se podría alcanzar.

La calificación de países según indicadores de eficiencia de sus sistemas de salud está influida de manera directa por las variables que se utilicen para medir tanto los productos como los insumos. Los países que tienen sistemas de salud eficientes son poco sensibles a cambios en los modelos para determinar la eficiencia. Para el resto de los países, un cambio en los modelos utilizados para medir eficiencia podría colocarlos en un lugar por encima o por debajo de su situación inicial.

Bajo rendimientos variables a escala, es posible que aquellos países que no tienen los mayores incrementos marginales en función de sus variables dependientes puedan ser parte de la frontera de mejor práctica, hecho que no está permitido bajo rendimientos no crecientes a escala. Sin embargo, el incremento en eficiencia de la mayor parte de los países estaría dado por el relajamiento del supuesto de disposición fuerte de insumos, por lo que este grupo de países podría mantener sus resultados de salud disminuyendo los insumos que se emplean para producirlos.

En los modelos que consideraron rendimientos no crecientes y variables a escala se encontraron países en los que, a pesar de tener altos ingresos, sus sistemas de salud tienen resultados muy deficientes en la esperanza de vida y la mortalidad infantil. La 
explicación a lo anterior se puede hipotetizar por la existencia de un umbral por debajo del cual el gasto en salud y el ingreso per cápita inciden de manera significativa sobre estos indicadores de resultado. Sin embargo, una vez rebasado ese umbral, estos indicadores sufren cambios no significativos y cada mejora tiene un costo que no resulta eficiente. También se debe tomar en cuenta que altas inversiones en salud inciden en otros indicadores no considerados como productos del sistema de salud, como es el caso de las enfermedades características de los países desarrollados (crónico-degenerativas).

Invertir una mayor proporción del PIB en los sistemas de salud no garantiza obtener mejores resultados si no se controla también la forma en que se invierten esos recursos. Se requiere hacer más eficiente el sistema de salud mediante los incentivos o mecanismos de regulación apropiados. Existe evidencia de que la cultura organizacional está ligada en forma contingente al desempeño de los sistemas de salud (Mannion et al., 2003).

Es importante continuar trabajando sobre el tema de la eficiencia y la calificación de los sistemas de salud en diferentes aspectos para extender el conocimiento, ya que la metodología utilizada por la oms no es concluyente debido, entre otros factores, a la complejidad de la producción de salud y a la dificultad para la definición, operacionalización y medición de las variables involucradas y la heterogeneidad entre las naciones (Greene, 2004; Navarro, 2002). El presente artículo difiere de los mencionados con anterioridad en algunos aspectos como son: variables de insumo y producto, periodo de análisis, método de cálculo y supuestos. En este estudio, los resultados de eficiencia fueron sensibles a las variables incluidas para medir insumos y productos, así como a los supuestos del modelo. 
Anexo

Clasificación de países por nivel de ingresos

\begin{tabular}{|c|c|}
\hline Ingreso bajo & Ingreso medio-bajo \\
\hline Afganistán & Albania \\
\hline Angola & Argelia \\
\hline Bangladesh & Armenia \\
\hline Benin & Azerbaiyán \\
\hline Bután & Bolivia \\
\hline Burkina Faso & Bosnia y Herzegovina \\
\hline Burundi & Cabo Verde \\
\hline Cambodia & China \\
\hline Camerún & Costa de Marfil \\
\hline Chad & Cuba \\
\hline Comoras & Ecuador \\
\hline Congo & Egipto \\
\hline Djibouti & El Salvador \\
\hline Eritrea & Ex República Yugoslava de Macedonia \\
\hline Etiopía & Georgia \\
\hline Gambia & Guatemala \\
\hline Ghana & Guyana \\
\hline Guinea & Honduras \\
\hline Guinea-Bissau & Indonesia \\
\hline Haití & Irak \\
\hline India & Islas Marshall \\
\hline Kenya & Islas Salomón \\
\hline Kiribati & Jamaica \\
\hline Madagascar & Jordania \\
\hline Malawi & Kirguistán \\
\hline Mali & Lesotho \\
\hline Mauritania & Liberia \\
\hline Mozambique & Maldivas \\
\hline Myanmar & Marruecos \\
\hline Nepal & Micronesia (Estados Federados de) \\
\hline Níger & Mongolia \\
\hline Nigeria & Namibia \\
\hline República Centroafricana & Nicaragua \\
\hline República Democrática del Congo & Pakistán \\
\hline República Democrática Popular Lao & Papua Nueva Guinea \\
\hline República Popular Democrática de Corea & Paraguay \\
\hline República Unida de Tanzania & Perú \\
\hline Ruanda & República de Moldova \\
\hline Santo Tomé y Príncipe & Samoa \\
\hline Senegal & Sri Lanka \\
\hline Sierra Leona & Suriname \\
\hline Somalia & Tonga \\
\hline Sudán & Ucrania \\
\hline Tayikistán & Uzbekistán \\
\hline Togo & Vanuatu \\
\hline Uganda & Vietnam \\
\hline Yemen & Zimbawe \\
\hline Zambia & \\
\hline
\end{tabular}


Anexo (continúa)

\begin{tabular}{|c|c|}
\hline Ingreso medio & Ingreso alto \\
\hline Antigua y Barbuda & Alemania \\
\hline Argentina & Andorra \\
\hline Belarús & Arabia Saudita \\
\hline Belice & Australia \\
\hline Botswana & Austria \\
\hline Brasil & Bahamas \\
\hline Bulgaria & Bahrein \\
\hline Chile & Barbados \\
\hline Chipre & Bélgica \\
\hline Colombia & Brunei Darussalam \\
\hline Costa Rica & Canadá \\
\hline Croacia & Dinamarca \\
\hline Dominica & Eslovenia \\
\hline Estonia & Emiratos Árabes Unidos \\
\hline Federación de Rusia & Eslovaquia \\
\hline Fiji & España \\
\hline Filipinas & Estados Unidos de América \\
\hline Gabón & Finlandia \\
\hline Granada & Francia \\
\hline Guinea Ecuatorial & Grecia \\
\hline Irán (República Islamica del) & Hungría \\
\hline Jamahiriya Árabe Libian & Irlanda \\
\hline Kazajstán & Islandia \\
\hline Letonia & Islas Cook \\
\hline Líbano & Israel \\
\hline Lituania & Italia \\
\hline Malasia & Japón \\
\hline Malta & Kuwait \\
\hline Mauricio & Luxemburgo \\
\hline México & Mónaco \\
\hline Omán & Nauru \\
\hline Palau & Niue \\
\hline Panamá & Noruega \\
\hline Polonia & Nueva Zelanda \\
\hline República Àrabe Siria & Países Bajos \\
\hline República Dominicana & Portugal \\
\hline Rumania & Qatar \\
\hline San Vicente y las Granadinas & Reino Unido de Gran Bretaña e \\
\hline Santa Lucía & Irlanda del Norte \\
\hline Serbia y Montenegro & República Checa \\
\hline Sudáfrica & República de Corea \\
\hline Swazilandia & Saint Kitts y Nevis \\
\hline Tailandia & San Marino \\
\hline Trinidad y Tobago & Seychelles \\
\hline Túnez & Singapur \\
\hline Turkmenistán & Suecia \\
\hline Turquía & Suiza \\
\hline Uruguay & Tuvalu \\
\hline Venezuela & \\
\hline
\end{tabular}

Fuente: Elaboración propia con base en información de la oms (WHOsis, 2005). 


\section{Bibliografía}

Babazono, Akira y Alan Hillman (1994), "A comparison of international health outcomes and health care spending", Int J Technol. Assess. Health Care,10 (3): 376-381.

Bartley, Mel (2004), Health Inequality, Blackwell Publishing, Oxford, Reino Unido.

Bitran, Ricardo y Steven Block (1992), Provider Incentives and Productive Efficiency in Government Health Services, Research Paper 1, Health Financing and Sustainability Project.

Cooper, William, Lawrence Seiford y Kaoru Tone (2000), Data Envelopment Analysis: A Comprehensive Text with Models, Applications, Reference and DEA-Solver Software, Kluwer Academic Publishers, Países Bajos.

Evans David, Ajay Tandon, Murray Christopher, Lauer Jeremy (2001), "Comparative Efficiency of National Health Systems: Cross National Econometric Analysis”, BMJ, 323: 307-310.

Fare, Rolf, Shawna Grosskopf, Mary Norris y Zhongyang Shang (1994), "Productivity Growth, Technical Progress, and Efficiency Change in Industrialized Countries", The American Economic Review, 84: 66-83.

Filmer, Deon y Lant Pritchett (1999), “The Impact of Public Spending on Health: Does Money matter?", Social Science and Medicine, 49 (10): 1309-1323.

Gravelle, Hugh, Rowena Jacobs, Andrew Jones y Andrew Street (2002), Comparing the Eficiency of National Health Systems. A Sensitivity Analysis of the WHO Approach, The University of York, Centre for Health Economics.

Greene, William (2004), “Distinguishing between Heterogeneity and Inefficiency: Stochastic Frontier Analysis of the World Health Organization's Panel Data on National Health Care Systems", Health Economics, 13: 959-980. 
Halstead, Scott, Julia Walsh y Kenneth Warren (eds.) (1985), Good Health at Low Cost, Rockefeller Foundation, Nueva York.

Hsiao, William (2000), What should Macroeconomists know about Health Care Policy? A Primer, IMF Working Paper, International Monetary Fund, Washington, D.C.

Mannion, Russell, Huw Davies y Martin Marshall (2003), Evidence on the Relationships Between Organisational Culture and Organisational performance in the NHS. CHE Draft 1, junio, The University of York, Centre for Health Economics, Reino Unido.

Mathers, Colin, Ritu Sadana, Joshua Salomón, Christopher Murray y Alan López. (2000), Estimates of DALE for 191 Countries: Methods and Results, World Health Organization, Ginebra.

Mellor, Jennifer y Jeffrey Milyo (2001), "Re-examining the Evidence of an Ecological Association between Income Inequality and Health", Journal of Health Politics, Policy, and Law, 26 (3): 487-522.

Murray, Christopher y Julio Frenk (1999), A wHO Framework for Health System Performance Assessment, World Health Organization, Ginebra.

Navarro, Vicente (2002), "Can Health Care Systems Be Compared Using a Single Measure of Performance?", American Journal of Public Health, 92 (1): 31-34.

Oxley, Howard y Maitland MacFarlan (1994), Health Care Reform: Controlling Spending and Increasing Efficiency, Organization for Economic Co-Operation and Development, Economics Department, Working Papers 149, París.

Palmer, Stephen y David Torgerson (1999), "Definitions of efficiency", British Medical Journal, 318 (7187):1136, marzo, documento pdf disponible en <http://www.bmj.com/ cgi/reprint/318/7191/1136.pdf $>$. 
Retzlaff-Roberts, Donna Cyril, F. Chang y Rose M. Rubin (2004), "Technical Efficiency in the Use of Health Care Resources: A Comparison of OECD Countries", Health Policy, 69: 55-72.

Smith, Peter (2002), "Composite Indicators of Health System Performance", en Peter Smith (ed.), Measuring up: Improving Health Systems Performance in OECD Countries, OECD, París, pp. 295-316.

Tangcharoensathien,Viroj y Jongkol Lertiendumrong (2000), "Health-System Performance", The Lancet, 356 (supplement 1): S31.

wHO (World Health Organization) (2000), World Health Report. World Health, Ginebra.

wHO (World Health Organization) (2002), World Health Report, World Health, Ginebra.

WHOSIS (wHO Statistical Information System) (2005), Core Health Indicators, documento html disponible en <http:// www.who.int/whosis/database/core/core_select.cfm, [consulta: enero-2005].

Wilkinson, Richard G., y Kate E. Pickett (2006), "Income Inequality and Population Health: A Review and Explanation of the Evidence", Social Science and Medicine, 62: 1768-1784.

Recibido: 25 de noviembre de 2005. Reenviado: 28 de septiembre de 2006. Aprobado: 26 de octubre de 2006.

Armando Nevárez Sida es maestro en economía por el Centro de Investigación y Docencia Económicas (CIDE). Actualmente se desempeña como investigador asociado B en el Instituto Mexicano del Seguro Social. Sus principales líneas de investigación son evaluación económica en salud, crecimiento económico y evasión fiscal. Entres sus publicaciones destacan: " $¿ E v a d i r$ o pagar impuestos? Una aproximación a los mecanismos sociales del cumplimiento" (en coautoría con Marcelo Bergman), Política y Gobierno, XII (1): 9-40 (2005); "Do Audits Enhance Compliance? An Empirical Assessment of VAT Enforcement" (en coautoría con 
Marcelo Bergman), Nacional Tax Journal, 2006 Dec., 59 (4): $753-$ 75; "Cost-effectiveness of Chronic Hepatitis C Treatment with Thymosin Alpha-1" (en coautoría con Fernando M. García Contreras, Patricia Constantino-Casas, Abud Bastida y Juan Garduño Espinoza), Archives of Medical Research, 37(5): 663-673 (2006).

Patricia Constantino Casas es maestra en Economía de la Salud en el Centro de Investigación y Docencia Económicas (CIDE) y MPhil en Health Sciences and Clinical Evaluation en la Universidad de York, Reino Unido. Actualmente se desempeña como investigadora de la Unidad de Investigación en Economía de la Salud, IMSS, y es miembro de la International Society for Pharmacoeconomics and Outcomes Research. Su línea de investigación actual corresponde a evaluaciones económicas de tecnologías para la salud, farmacoeconomía, determinantes y característica de la oferta y demanda de atención médica (polifarmacia, maltrato en el anciano, desigualdad y bajo peso al nacer, barreras de accesibilidad-cita previa, incapacidad para el trabajo, continuidad y calidad en la atención médica). Entre sus publicaciones como coautora destacan: Socioeconomic factors and low birth weight in Mexico (en coautoría con Laura P. Torres Arreola, Sergio Flores Hernández, Juan Pablo Villa Barragán y Enrique Rendón Macías), BMC Public Health, 5: 20, (2005), documento en html disponible en <http:// www.biomedcentral.com/1471-2458/5/20>; "Direct Medical Costs for Partial Refractory Epilepsy in Mexico", Archives of Medical Research, 37 (3): 376-83, (2006); "Estudio de las necesidades de salud en atención primaria a través del diagnóstico de salud de la familia", Aten Primaria, 38 (7): 383-388 (2006); "Prescripción de certificados de incapacidad temporal en el IMSs. Perspectiva desde la economía de la salud", Revista Medica del Instituto Mexicano del Seguro Social, 45 (1): 89-96 (2007).

Fernando García Contreras es maestro y candidado a doctor en ciencias médicas. Actualmente se desempeña como investigador en la Unidad de Investigación en Economía de la Salud, Instituto Mexicano del Seguro Social y como profesor de farmacoeconomía, Universidad Autónoma del Estado de Morelos. Su línea de investigación se centra en farmacoeconomía y economía de la salud. Entre sus publicaciones destacan: "Cost-effectiveness of chronic hepatitis C Treatment with thymosin alpha-1" (en coauto- 
ría con Armando Nevárez Sida, Patricia Constantino Casas, Abud Bastida y Garduño Espinoza), Archives of Medical Research, 37(5): 663-673 (2006); “Direct Medical Costs for Partial Refractory Epilepsy in Mexico" (en coautoría con Patricia Constantino-Casas et al., [Journal Article, Multicenter Study] Archives of Medical Research, 37(3): 376-83 (2006). 